\title{
Hybrid TDR-MI Based Wireless Sensor Network for Underground Water Pipeline Leakage Detection and Localization Using Pressure Residuals and Classifiers.
}

\section{Ramdas Vankdothu ( $\sim$ vramdas786sap@gmail.com )}

Osmania University University College of Engineering https://orcid.org/0000-0002-8478-1291

Hanumanthu Bhukya

Kakatiya University

Raghu Ram Bhukya

KITS: Kakatiya Institute of Technology and Science

\section{Research Article}

Keywords: Time Domain Reflectometry, Magnetic Induction, MISE PIPE, Ad-hoc sensor network, K-means clustering, Pipeline leakage detection and leak localization.

Posted Date: September 21st, 2021

DOl: https://doi.org/10.21203/rs.3.rs-788366/v1

License: (c) (1) This work is licensed under a Creative Commons Attribution 4.0 International License.

Read Full License 


\title{
Hybrid TDR-MI based Wireless Sensor Network for underground water pipeline leakage detection and localization using pressure residuals and classifiers
}

\author{
A.Ramdas Vankdothu, B. Hanumanthu Bhukya, C. Raghu Ram Bhukya,
}

\begin{abstract}
The pipeline leakage detection and leak localization trouble is a highly demanding and dangerous issue. Underground pipelines are critical for transporting enormous fluid volumes (e.g., water) across extended distances. Solving this problem will save the country much money and resources, but it will also protect the environment. On the other hand, present leak detection technologies are insufficient for monitoring underground pipelines due to the extreme subterranean environmental conditions. This study proposes a hybrid wireless sensor network based on TDR (time domain reflectometry) and magnetic induction for monitoring underground pipelines to solve these problems. In this scenario, TDR is deployed beneath an MI-based wireless sensor network. TDR precisely locates the leak and dramatically decreases the amount of time required for inspection. We offer a wireless sensor network for low-cost, real-time leak detection in subsurface pipes based on MI technology. MISEPIPE identifies leaks by integrating data from different types of sensors installed within and around underground pipelines. Adhoc WSNs are used to measure pressure. (WDNs) is a hot topic that has piqued researchers' interest in recent years. Time and accuracy are critical components of leak localization, as they substantially impact the human population and economy. Statistical classifiers acting in the residual space are offered as a general method for leak localization. Classifiers are trained on leak data from all network nodes, taking demand uncertainty, sensor preservative noise, and leak magnitude on the account. Following leak identification and localization, all monitoring data is forwarded to the $\mathrm{CH}$ using the K-means clustering method, which serves two critical functions: optimal clustering, prolonging the Network Lifetime (NL), and preserving the QoS. The clustering method is optimized using the K-Means approach.
\end{abstract}

Index Terms-Time Domain Reflectometry, Magnetic Induction, MISE PIPE, Ad-hoc sensor network, K-means clustering, Pipeline leakage detection and leak localization.

\section{INTRODUCTION}

Wireless sensor networks or Remote sensor systems for pipeline spillage recognition rely on the liquid for the most part. The pipeline is shipping just as the earth in which the pipeline is set or installed. Remote intelligent sensor systems are a viable option for monitoring the condition of covered water pipes, specifically their weight and, subsequently, spills. Their advantage over other frequently used break locating systems is that they incorporate an element of excess, as individual damaged hubs do not render the entire framework obsolete and allow for continuous monitoring without administrator intervention. Remote sensor Systems with ultra-low force sensitivity enable them to operate independently for extended periods [1]. Water gracefully pipelines assume a significant job in the foundation of urban communities and processing plants. As it may, pipelines are vulnerable to spill because of such factors as nature, maturing pipe, outer powers, and erosion [2]. Water Distribution Systems (WDS) convey new water from flexible sources and capacity stores/tanks to modern, business, and local locations through a mind-boggling web of pipeline frameworks. In any case, new water supplies proceed to diminish, and $66 \%$ of the world will encounter water pressure, and about $25 \%$ will encounter degraded water shortage [3]. Due to the difficulties of WDN, numerous strategies and methodologies have been developed with an emphasis on precision and efficacy.

Leakage detection technologies or Spillage location innovations have been becoming exceptionally quick over the most recent two decades. They offer monetary reserve funds, liquid deficiency decrease, improve utilization, and lessen pipeline arrange harms. Typically, complete discovery extracts highlights from commotion-disrupted signals and does AI on signal properties. For instance, the weight wave highlight vector was isolated and used to contribute to the least squares support vector machine to differentiate pipeline spills. [4] [5]. A funnelling appropriation system investigating courses through the pipelines, including foundation spillage streams, should dramatically advance identifying such spillages [6]. Several strategies have been proposed for spillage discovery. These strategies can be partitioned into two primary classes called the equipment and programming based techniques. Equipment based techniques utilize uncommon sensors to straightforwardly recognize the event of the spillage and decide its area. Then again, programming-based strategies use the standard sensors implanted in the pipeline Data Acquisition and Supervisory Control framework [7]. Leakage in the significant distance pipelines may prompt extreme debilitation to water

A. Ramdas Vankdothu is pursuing his Doctoral degree in Computer Science \& Engineering at Osmania University Hyderabad, India (e-mail: vramdas786sap@gmail.com).

B. Hanumanthu Bhukya, Assistant Professor in KITS and pursuing his Doctoral degree in Computer Science \& Engineering at Kakatiya University Warangal, India.

C..Raghu Ram Bhukya, Assistant Professor in Department of Computer Science, KITS, Warangal, India. 
transportation. Managing the information gathered by remote sensor systems (WSNs) is fundamental to accomplish ongoing spillage restrictions to diminish water misfortune. Additional sensors may increase the adequacy of monitoring leakage from long-distance pipelines [8].

Localization of the source of the leak Confinement of the release via the negative weight wave approach is time dependant on the time required for a negative constrain wave to reach the pipeline's two closures. The harmful weight waves generated by a hole are connected to the pipeline's operating circumstances, length, and diameter, as well as the liquid's properties. Regardless, the awareness of negative weight waves directly affects the technique's ability to contain the hole [9]. Leak prevention is a critical aspect of funnel failure detection with remote sensor systems. The mean power of a particular separation was determined using a vitality-based confinement strategy that separates the release locations based on assessments of the separations between realized estimation focuses and hidden break. The standard deviation and change were resolved. To highlight the link, the straight relapse is used. Following that, the force values from the actual pipeline spill precisely separate the sensor hubs from the hole and approve the relationship details [10] [11].

\section{LITERATURE SURVEY}

Haoran Zhang et al. [12] have introduced a hybrid strategy for leak identification and identification for fluid pipelines dependent on the backward water driven and thermodynamic transient examination technique and the improved PSO calculation. The primary objective of this examination is to suggest a hole finding and limiting strategy for fluid pipelines based on comparative water-powered thermodynamic transient investigations and upgraded particle swarm optimization (PSO). The limited volume technique was used to mathematically represent the conditions of coherence, force, and life. Four different computations investigated a virtual pipeline to determine the optimal enhanced PSO calculation and compare boundaries. The tests for precision, solidity, strength, and bogus caution rates were directed. SIPSO computations have been shown to outperform other analyses.

Dalei $\mathrm{Wu}$ et al. [13] have implemented an SN self-localization calculation into an automated sensor arrangement to assess subsurface water pipelines. The purpose of this analysis is to track the position and speed of an SN inside a funnel using estimates of both the SN's speed and the radio's RSS from over-the-ground RNs. In light of the automated sensor organization's unique engineering framework models included SN elements and estimation models.

Chao-Chih Lin et al. [14] another transient-based cross breed heuristic methodology has been devised to improve the transient age process and detect pipe system spills. The methodology addresses the development issue by integrating the ordinal development approach (OOA) and searching for symbiotic organisms (SOS). The consistent state head dissemination and channel stream rates are determined using a pipe network analysis model (PNSOS). By increasing the target capacity of transitory vitality, the optimal transient age point and critical valve activity boundaries are strengthened. A transient event is generated at the designated location, and the transient stream is decomposed using the typical approach (MOC). When spill data is available, the OOA filters out competing pipes and underlying living organisms. The SOS technique detects holes by reducing the total amount of contrasts between reproduced and figured heads at perception focuses. Two fictitious release scenarios are used to demonstrate the leak detection ordinal symbiotic organism search: a simple funnel configuration and a water distribution network (WDN) (LDOSOS).In both cases, the offered approach correctly identifies the Leak data.

LiseFerrandez-Gamot et al. [15] proposed another technique for defect localization devised to use models, pressure sensors, and classifiers in combination. The water-powered model of the system is utilized to create residuals by comparing model expectations to sensor-provided estimations. After generating residuals, they are dissected using managed classifiers to facilitate leak localization. The classifiers are adjusted utilizing information in spill situations in all the system's hubs, thinking about vulnerability sought after appropriation, added substance clamour in sensors and vulnerability in spill size.

Xue-yuanwang et al. [16] was provided to develop an SVM model that used particle swarm optimization (PSO) to determine the two uncertain borders. Particle swarm optimization is a wise computational multiplicity optimization technique that advances an issue by attempting to better an applicant arrangement by a predetermined percentage. The approach possesses a high capability for global pursuit, but it is also quite effective at implementation. The PSO computation is used to advance the SVM model's boundaries to improve its precision. After assembling, preparing, testing, and classifying the example set, a forecast model for water pipeline spill discovery is created using PSO-SVM.

ZiguangJia et al. [17] To locate leakages, an SVR-based pattern recognition technique was introduced. It is demonstrated that by combining applicable circle strain detecting data with help vector relapse (SVR), the location of spillage on a considerable distance pressurized pipeline can be pinpointed. Additionally, the SVR limits were simplified using a genetic algorithm (GA) to improve their precision. 


\section{PROBLEM STATEMENT}

Existing studies have focused on the leak detection problem and the number of leaks in their location. The problem is highly challenging due to the following limitations of the environment:

1. The investigation of estimations to not choose the most reasonable period for leakage identification.

2. On the other hand, the localization of the best estimation focuses on an erroneous examination, and leak detection is recognized inaccurately.

\section{PROPOSED METHODOLOGY}

In this research, a novel approach for underground pipeline monitoring is presented: Hybrid TDR (time domain reflectometry)-magnetic induction-based wireless sensor networks

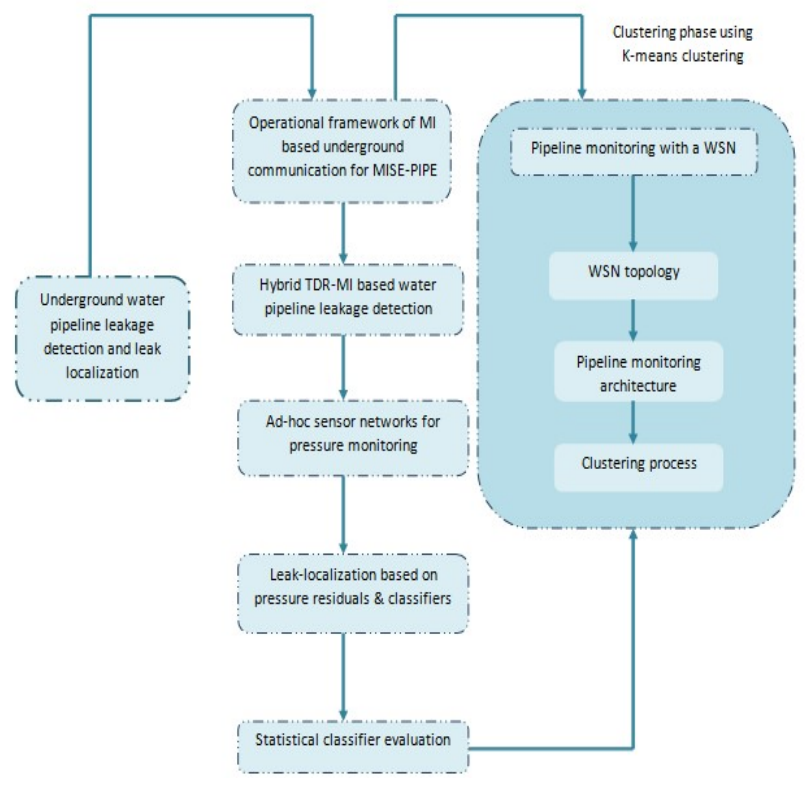

Figure 1: Overall Block diagram of the proposed methodology.

. TDR is installed beneath an MI-based wireless sensor network in this example. TDR pinpoints the leak not only exactly but also significantly reduces the inspection time required. We offer a wireless sensor network for low-cost, real-time leak detection in subsurface pipes based on MI technology. MISE-PIPE identifies leaks by integrating data from a range of different types of sensors installed within and around underground pipelines. Ad-hoc WSNs are used to measure pressure. Following that, pipeline leak localization in water distribution networks (WDNs) is a hot topic that has piqued researchers' interest in recent years. Time and accuracy are critical components of leak localization, as they substantially impact the human population and economy. Due to the difficulties of WDN, numerous strategies and methodologies have been developed with an emphasis on precision and efficacy. Statistical classifiers acting in the residual space are offered as a general method for leak localization. Classifiers are trained on leak data from all network nodes, taking demand uncertainty, sensor preservative noise, and leak magnitude on the account. Following leak identification and localization, all monitoring data is forwarded to the $\mathrm{CH}$ using the $\mathrm{K}$-means clustering method, which serves two critical functions: optimal clustering, prolonging the Network Lifetime (NL), and preserving the QoS. The clustering method is optimized using the K-Means approach.

The overall process of the proposed methodology is diagrammatically given in figure 1 . The concept includes the whole process is explained in detail as follows;

\subsection{Underground water pipeline Leakage detection:}

Underground water pipeline Leakage identification advances have been becoming extremely quick over the most recent two decades. They offer financial investment funds, liquid deficiency decrease, and improve utilization and lessen pipeline network harms. Leakage that is Spillage can be distinguished by recognizing material trickle out at the particular spot. Sometimes, spillage might be watched, yet the area isn't known due to the wide conveyance of the channel arrangement. The likelihood of the framework debasement gets higher as the framework gets more extensive, so keeping up the framework persistently ensures an ideal and safe framework. The fundamental function of Leakage Detection Systems (LDS Systems) is to help pipeline controllers in discovering and limiting breaks.LDS Systems play a critical role in pipeline innovation. There are four primary leakage reduction exercises: exerting pressure on executives, dynamic whole-system control, implementing speed and quality 
solutions, and supporting and reestablishing pipelines. Old detection strategies rely upon the periodical review led by the upkeep group, which has numerous detriments, for example,

- Does not provide real-time pipeline monitoring.

- Cause a much larger financial defeat.

- Cause ecological contamination.

An underground wired system for correspondence experiences harms, and it is exorbitant. Remote systems, then again, are considerably more powerful and effective. It also gives adaptability and basic framework sending, yet underground, remote correspondences still can't seem to be created and figured out. Be that as it may, Wired base communication can transmit data over long distances to reach remote business hubs.

\subsection{Operational structure of magnetic induction based subsurface communication for MISE-PIPE:}

The magnetic induction method is used to give productive and powerful remote correspondences for the underground sensors. MI-based remote sensor arranges minimal effort answer for constant checking framework for leakage detection. For small underground interchanges, Electro-Magnetic (EM) waves experience the ill effects of spread constriction, yet attractive felid doesn't.MI attenuation is directly proportional to $\frac{1}{r}$, while EM wave's attenuation is directly proportional to $\frac{1}{r^{3}}$ (where $\mathrm{r}$ : distance). Also, MI channels do not vary as the soil property changes. MI communication is carried out on pipelines using a loop of wire panting for shortness of breath. The sign in the transmitter curl is controlled by a sinusoidal current, which generates a period-varying attractive field that initiates another sinusoidal current in the authority circle, which accomplishes the underground MI remote correspondence as demonstrated in figure 2. This procedure uses an MI waveguide that joins a few hand-off loops to develop a strong and beneficial confidential far-off correspondence. Move collies are passed on at any path for every 6-12 meters. MI-based includes two kinds of course of action, for instance, inside or outside the pipeline.

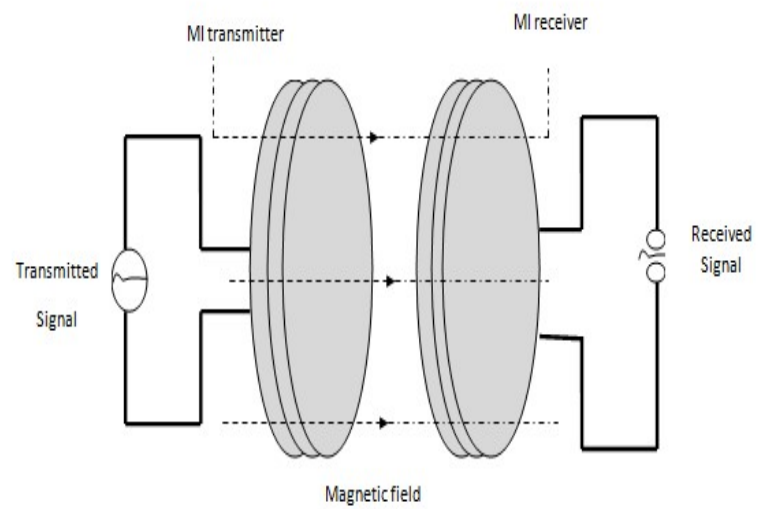

Figure 2: magnetic induction-based wireless communication

Outside and inside pipeline based monitoring techniques:

Outside based monitoring techniques: If the liquid is fluid, a soil moisture sensor can precisely determine leaks outside of funnels. Similarly, a temperature sensor is used to detect hot substances that have been spilled. The subterranean sensors are coupled to an above-ground reception device for long-distance remote communication.

Inside pipeline based monitoring techniques: This strategy involves introducing sensors into the pipeline via syphon stations and checkpoints. When a small amount of leakage occurs, the channel divider produces high repetition vibrations that can be detected using acoustic transducers. Because acoustic sensors can detect only minute leakage, they are most effective when utilized close to pump stations and checkpoints.

\subsection{Hybrid TDR (time domain reflectometry)-magnetic induction based system structure:}

Our investigation established the use of TDR framework to identify spills, or leakage locations, in subterranean pipes. A biwire is added in parallel with the subsurface MISE pipes and is used as a TDR sensor in this architecture. This technology overcomes significant limitations associated with the standard methods for locating acoustic leaks. The break identification approach is moderately precise when the TDR sensor is in touch with water in only one point. TDR is attached to the bottom of the MISE-PIPE-based remote sensor organization. 
TDR pinpoints the break not only precisely but also significantly reduces the time required for the examination. The framework used in this case is a clustered architecture composed of heterogeneous sensors. Each pipeline segment contributes to the formation of the group. To extend the lifetime of the framework, all sensors' power consumption must be limited, as batteries power them. The sensors are separated into the central point layer (also called the hub layer) and the soil layer. The center point sensor is installed internally to the pipeline and comprises weight and acoustic sensors situated at checkpoints or siphon stations. The center sensors interact with the dirt property sensors via appealing enlistment devices. The dirt layer has sensors for determining soil parameters outside the pipeline, such as moisture and temperature sensors. Estimates were transmitted remotely to the cluster head at the checkpoint or siphon station using a hybrid TDR and MI technique. Then the cluster head group collated the data and transferred it remotely through the air, EM waves, to a remote organization location. Figure 3 illustrates the framework structure.

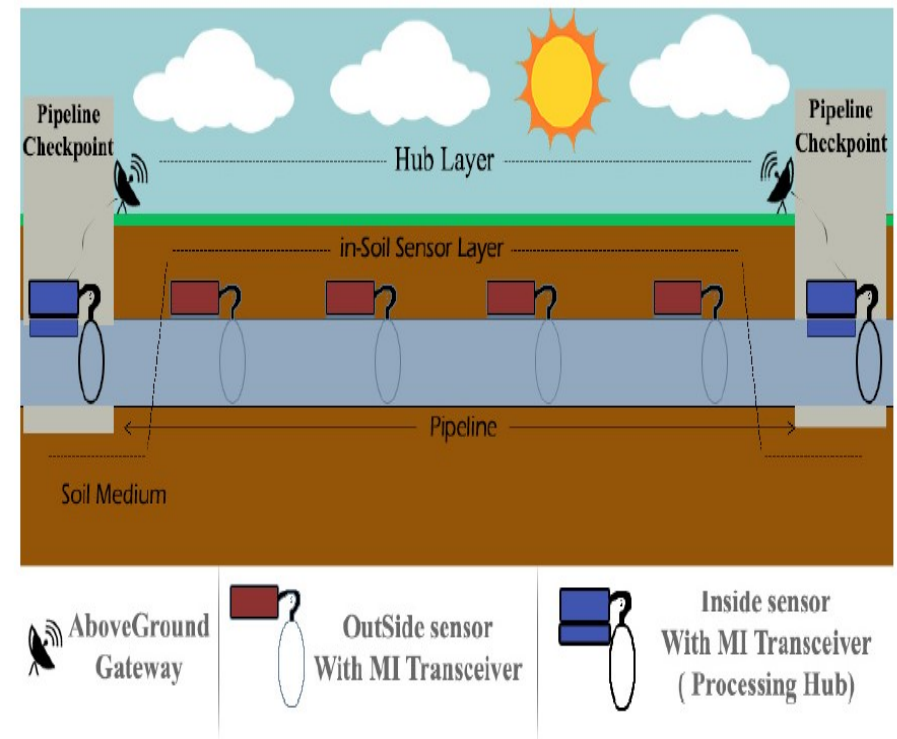

Figure 3: system structure of Hybrid TDR (time domain reflectometry)-magnetic induction

The above figure 3 clarifies the framework structure of Hybrid TDR_magnetic induction. The following sections detail the hybrid TDR magnetic induction schematization of the standard format of an underground funnel equipped with a scattered biwire:

The TDR approach is based on transmitting an electromagnetic pulse and measuring the delay time required for a portion of the signal to be reflected to the source point. This pulse is a voltage stage signal with a rapid climb time conveyed by the sensor and into the test condition. The reflected signal contains a wealth of information about the materials' dielectric characteristics surrounding the sensor. Underground water pipeline furnished with the dispersed bi-wire. The idea incorporate this is clarified point by point as follows;

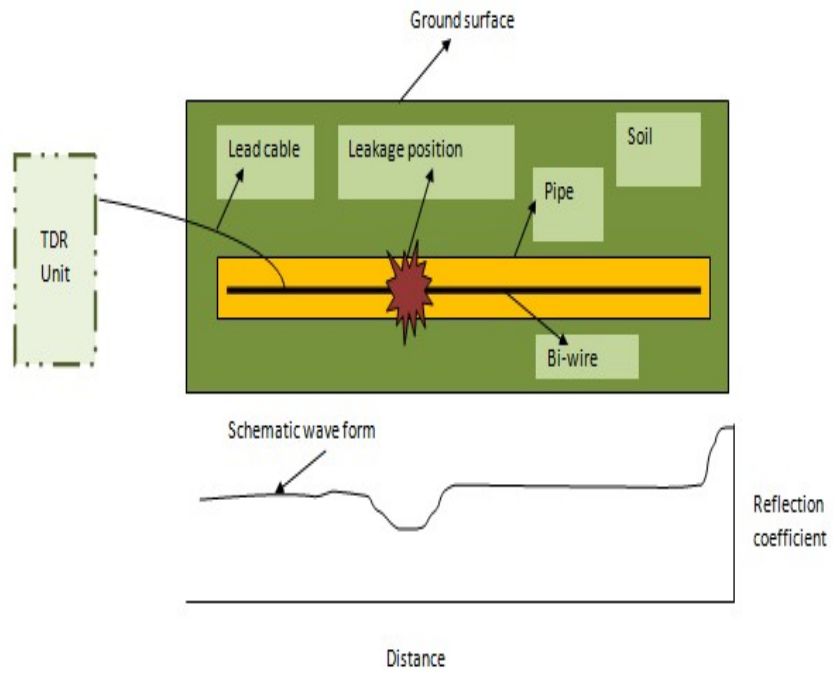

Figure 4: MISE pipe equipped with the distributed bi-wire 
In TDR estimations, the proportion among the plentifulness of the sign that is reflected by the framework under test (UR) as well as the adequacy of the created signal (UI) will be shown as reflection coefficient (q).

$$
q=\frac{U R}{U I}
$$

The primary advantage of TDR is that the reflection position and its causes are determined independently of the transmission term and reflection qualities. The dielectric constant of the materials that surround the bi-wire contributes to the electromagnetic wave transmission speed. This speed is typically expressed as a fraction of the speed of light in a vacuum, abbreviated VOP, which is one of the cable parameters. The accompanying condition indicates the methodologies of hybrid TDR-MI based remote sensor organization.

$$
\begin{aligned}
& Z=\left\{M I(M I P I P E)+B i \_ \text {Wired }\right\} \\
& U_{\text {cable }}=\frac{2 L}{T} \\
& U O R=\frac{2 L / T}{C} \\
& \varepsilon=\left(\frac{1}{U O R}\right)^{2}
\end{aligned}
$$

Anywhere, $\mathrm{L}$ denotes the link, which is the cable length (the use of $2 \mathrm{~L}$ is due to the sweep of the pulse over the sensor), $\mathrm{T}$ denotes the time required for the pulse to travel from the link's earliest starting point to the farthest point possible, $\mathrm{C}$ represents the light speed in a vacuum, as well as the dielectric constant of the materials enclosing the bi-wire.

One of the most significant boundaries of the link is the attributes impedance $\left(S_{0}\right)$, and the indication of electromagnetic pulses happens because of the absence of impedance coordinating. Additionally, the amount of reflection coefficient can be expressed numerically in terms of the electrical impedance condition. 6

$$
q=\frac{S_{1}(d)-S_{0}}{S_{1}(d)+S_{0}}
$$

Wherever, $S_{0}$ the trademark impedance of the transmission is the line, in addition, to $S_{1}$ be the heap impedance at separation d, and it relies upon the effective dielectric permittivity of the transmission line at the thought about segment.

For a bi-wire transmission line, the trademark impedance is a component of the geometry, the size, the separation among two channels, and the dielectric consistent of the protection isolating them from one another, which are numerically spoken to as the condition7.

$$
S_{0}=\frac{276}{\sqrt{k}} \log \frac{2 n}{D}
$$

Where $\mathrm{k}$ is the dielectric steady of protection, $\mathrm{m}$ is the distance between two channels, and $\mathrm{D}$ is the breadth of the transmitter. Thus, the appearance of an electromagnetic pulse occurs in the presence of a change in dielectric constant or a change in geometry. The area of these progressions is estimated using the conditions previously indicated. Thus, the perception of an electromagnetic pulse occurs in a dielectric change or a misshaped geometry. The area of these progressions is estimated using the conditions previously indicated. The framework for TDR-based leak detection violates the physical specifications for TDRbased material analysis. This method of detecting leaks is based on sensing the variation in dielectric properties in the dirt caused by water escaping from the funnel. The proximity of water (whose relative (dielectric) permittivity is approximately equal to 80 ) induces a discernible variation in the dielectric properties of the dirt (whose relative permittivity does not typically exceed 2-3 in dry conditions). 
When the TDR approach is used as a leakage detector, a bi-wire link is used to connect a TDR sensor to the channels, with the materials surrounding the sensor acting as the wave generating condition. Spillage water alters the electrical characteristics of the transmission line, which can be readily detected using TDR methods. The establishment and operation of the TDR are depicted schematically in Fig. 1. Following that is pressure monitoring. In this case, ad-hoc remote sensor systems are used. The concept of ad-hoc systems is explained in detail as follows:

\subsection{Ad-hoc wireless sensor networks for pressure monitoring:}

The utilization of ad-hoc remote sensor systems is one of the most current and feasible methods for detecting leaks in underground pipeline systems. WSNs in this framework make use of portable remote sensors that can detect cracks or holes in underground pipelines by checking or monitoring the pressure of liquid flowing through them. The sensors transfer data acquired from the channels to the surface via a distant association, which does not require physical contact with the surface. Some stations receive and process the signal on the surface. Following signal reception and preparation, the stations connect via a focal unit that serves as the primary association point. The focus unit procedure obtained by the sign enables a repeatable and straightforward identifiable proof of spilling in the event of an incident. Compared to alternative spillage detection solutions currently available on the market, this procedure is efficient, straightforward, and consumes little electricity.

The complete system is composed of ground stations located in permanent or moveable locations. The ground stations are equipped with mandatory receiving cables and are oriented toward the domain to communicate with the various sensors deployed throughout the pipeline network. These sensors are at the heart of the framework's capability for observation. They contain a hydrophone for detection and a radiofrequency transmitter for transmission. The sensor's progression is limited and stabilized along the way by water-powered deceptions. If there is a spillage in the channel, the sensor notifies the nearest ground station of its location. The sensor's position is determined, and the spilled area is determined at that point [13]. By monitoring the degree of liquid pressure, the proposed network technique enables the detection of pipeline leaks or breakage. Remote channels are used to send the recognized data. After this, restrict the break-in water appropriation arranges to utilize pressure residuals just as classifiers. Constrain sensors to be operated by a leak localization technique dependent on pressure models as well as classifiers. The idea to incorporate this leak localization is explained in detail as follows;

\subsection{Leak localization based on pressure residuals and classifier:}

For leak localization, a method based on statistical classifiers operating in the residual space is proposed. Pipeline spillages in water distribution networks (WDN) are a noticeable issue that has increased enthusiasm among scientists in the previous barely years. Time, distance, and precision assume a significant job in leak localization as it has a huge effect on the human populace and financial perspective. The multifaceted nature of WDN has briefly presented various strategies and techniques concentrating on the exactness and adequacy. Water utilities have a fundamental practice of partitioning the water distribution network (WDN) into small, unconnected regions called District Metered Areas (DMAs) to enable superior leak monitoring and pressure control. Flow and pressure sensors are used to monitor the gulfs, and additional pressure sensors are inserted inside. Leak localization techniques are based on the estimations made by a large number of inserted sensors. Pressure sensors are frequently selected overflow sensors due to their lower cost and ease of installation and maintenance. Technique for locating leaks that is dependent on the plan depicted in Fig. 5.

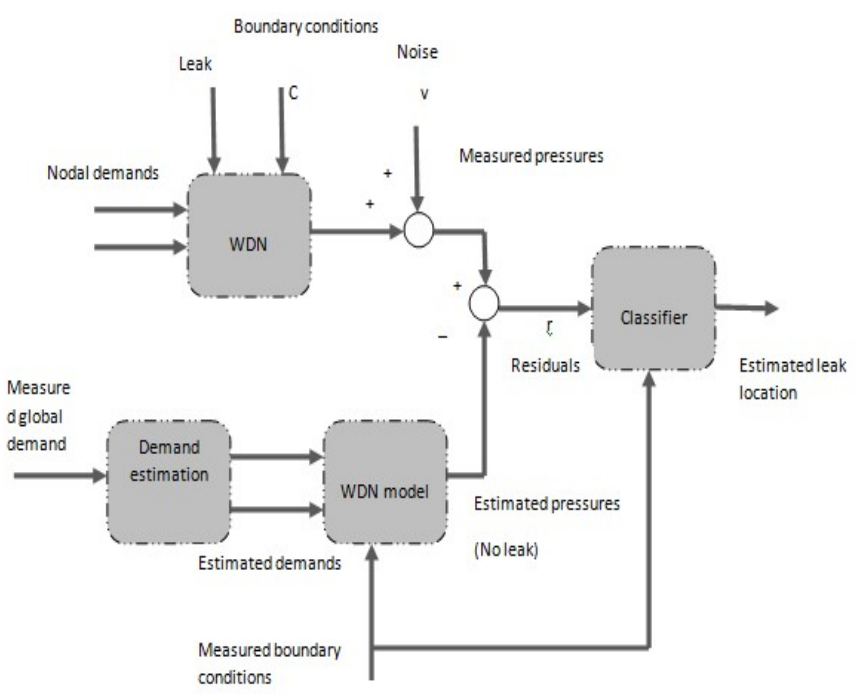

Figure 5: Leak localization scheme 
The above figure 5 which indicates the entire leak localization based on processing pressure residuals in addition to examining them by methods for a classifier. Residuals are processed as the contrasts among the estimations gave by pressure sensors. Leak localization techniques dependent on the classifiers are clarified as follows;

\subsection{Classifier Evaluation:}

Classifiers are developed based on leak data from all network hubs, considering uncertainty in command allocation, preservation noise inside sensors, and leak size. To evaluate the constructed classifier, one can build a disarray structure that summarizes the results obtained when the classifier is applied to an approved (or testing) informative collection. When applied to the problem of leak localization, the disarray lattice is a square grid with the same number of lines and segments as the classifier's classes, which is equivalent to the number of hubs or nodes in the system (potential leak locations), where each coefficient indicates how frequently a leak in node I is recognized as a leak in node j. Table 3 illustrates the chaos structure in action about leak localization.

In the event of an ideal classification, the disarray lattice ought to be slanting with $\Gamma_{i, j}=M_{v}$, intended for the entire $\mathrm{i}=1 \ldots$ $n_{c}$ being $M_{v}$ the size of a validation data set (number of examples in each class) and $n_{c}$ the number of classes. According to the preceding division, the quantity of classes $n_{c}$ is the number of places of WDN where a leak is measured, i.e. the number of network nodes $n_{n}$. Yet, this number could be changed, such as expelling the information from hubs near others and lessening the number of expected breaks (classes).

Table 1: Matrix $\Gamma$

\begin{tabular}{|c|ccccc|}
\hline & $\hat{l_{1}}$ & $\ldots$ & $\hat{l_{i}}$ & $\ldots$ & $\hat{l}_{n c}$ \\
\hline$l_{1}$ & $\Gamma_{1,1}$ & $\cdots$ & $\Gamma_{1, i}$ & $\ldots$ & $\Gamma_{1, c c}$ \\
$\cdot$ & $\cdot$ & $\cdot$ & $\cdot$ & $\cdot$ & $\cdot$ \\
$\cdot$ & $\cdot$ & $\cdot$ & $\cdot$ & $\cdot$ & $\cdot$ \\
$\cdot$ & $\cdot$ & $\cdot$ & $\cdot$ & $\cdot$ & $\cdot$ \\
$l_{i}$ & & & & & \\
$\cdot$ & $\Gamma_{i, 1}$ & $\ldots$ & $\Gamma_{i, i}$ & $\ldots$ & $\Gamma_{i, n c}$ \\
$\cdot$ & $\cdot$ & $\cdot$ & $\cdot$ & $\cdot$ & $\cdot$ \\
$\cdot$ & $\cdot$ & $\cdot$ & $\cdot$ & $\cdot$ & $\cdot$ \\
$l_{n c}$ & $\cdot$ & $\cdot$ & $\cdot$ & $\cdot$ & $\cdot$ \\
& $\Gamma_{n c, 1}$ & $\ldots$ & $\Gamma_{n c i}$ & $\ldots$ & $\Gamma_{n c x c}$ \\
\hline
\end{tabular}

Practically speaking, non-zero coefficients will show up outside the fundamental corner to corner of the matrix matrix $\Gamma$. Intended for a leak in node $\mathrm{i}$, the coefficient $\Gamma_{i, i}$ point out the number of times that the leak $l_{i}$ is properly recognized as $\hat{l}_{i}$, while $\sum_{j=1}^{n c} \Gamma_{i, j}-\Gamma_{i, i}$ point out the number of times that it is mistakenly classified. The general accuracy Ac of the classifier is characterized as

$$
\text { Accuracy }=\frac{\sum_{i=1}^{n c} \Gamma_{i, i}}{\sum_{i=1}^{n c} \sum_{j=1}^{n c} \Gamma_{i, j}}
$$

Because the precision esteem (1) only indicates the classification's goodness, not the accuracy of the leak localization, the Average Topological Distance (ATD) is employed to assess the whole execution. The ATD is a normal estimate of the base distance in hubs, defined as the nodes between the leaky hub and the hub applicant proposed by the leak localization technique. The ATD is processed as follows

$$
\text { Dis } \tan c e=\frac{\sum_{i=1}^{n c} \sum_{j=1}^{n c} \Gamma_{i, i} D_{i, j}}{\sum_{i=1}^{n c} \sum_{j=1}^{n c} \Gamma_{i, j}}
$$


Where $\mathrm{D}$ is a symmetric square lattice with size nc to such an extent that every component $D_{i, j}$ contains the base topological separation in hubs between the hubs alluded by $\mathrm{i}$ as well as $\mathrm{j}$. After the detection of pipeline leakage in addition to leak localization all the monitoring data information's are transmitted to the $\mathrm{CH}$ based on the K-means clustering algorithm, which explains detailed as follows;

\subsection{Clustering phase using K-means clustering Algorithm:}

\section{Pipeline monitoring with a WSN}

A.WSN topology: The process of finding a system, including its hubs and connecting links, is referred to as WSN geography. There are numerous topologies, including linear, point-to-point, bus, ring, star, and tree topologies. In this, we utilize natural geography due to its possible potential benefits in pipeline design, such as quick/cost proficient arrangement, decreased prerequisites for upkeep, expanded unwavering quality, and the capacity to effectively adjust multi-bounce steering conventions.

B. Pipeline monitoring Architecture: A structure for monitoring pipelines using WSNs is defined by many hubs, CHs, and a Base Station (BS). The clustering operation is carried out in this article utilizing Algorithm 2's point-by-point calculation. Sensor hubs monitor artificial boundaries throughout the pipeline such as pressure, flow, temperature, ph, conductivity, and turbidity. At that time, the checking information data is transmitted to the $\mathrm{CHs}$, who in turn sends it to a BS via one-jump or multiple-bounce correspondences, depending on the hub's configuration. For data exchange between sensor hubs, the checking systems use an intube correspondence mode. As shown in Fig. 6, the CHs are responsible for supervising sensor hubs within their cluster, combining pre-prepared data, applying information aggregation procedures, and sending it to the BS. The BS collects and transmits data from all $\mathrm{CHs}$ to the controller or end-user.

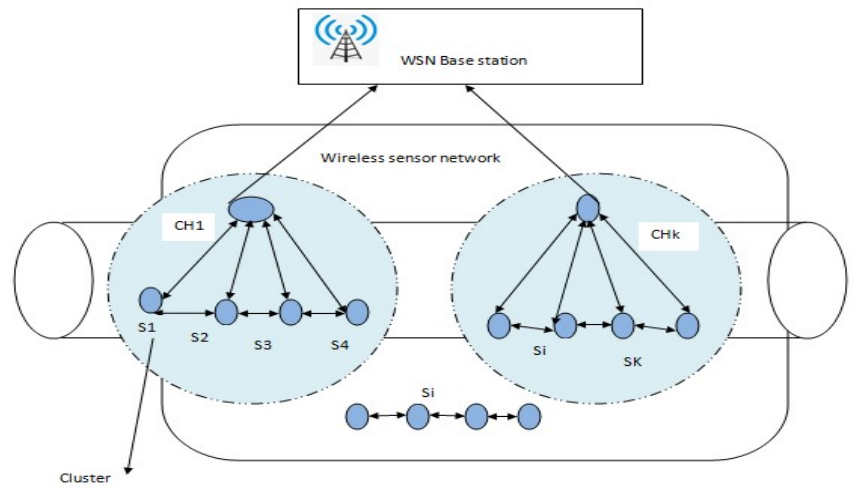

\section{Clustering Process:}

Figure 6: Pipeline monitoring scheme

Typically, clustering is used to create clusters inside WSNs. Regrettably, this approach has an issue with selecting the optimal CHs. This disadvantage motivated us to develop a new clustering approach known as K-Means. Indeed, the K-Means algorithm is a form of unsupervised learning frequently employed in network planning and can also tackle classification problems. This approach utilizes Euclidean distance to group nodes in our scenario. K-Means separates the data set (nodes) into k clusters based on their mean cluster values, reducing intercluster parallelism and increasing intra-cluster similarity. Technique 1 illustrates the stepladders used in this algorithm. The primary disadvantage of K-Means is its sensitivity to the pre-selection of CHs. This disadvantage prompted us to investigate a new variation of K-Means called K-Means++. By uniformly spreading the initial centers, the K-means++ method attempts to resolve the beyond crisis. Approach 2 describes this algorithm in depth.

\section{Algorithm 1: K-means}

Input: A set $S=\left\{s_{1}, s_{2}, \ldots s_{n}\right\}$ of the sensor as well as a number $\mathrm{k}(\mathrm{k}>1)$ of clusters to form

Output: A set of k clusters generated

Step 1: obtain $\mathrm{k}$ nodes randomly be in the right place to $\mathrm{S}$ as initial centroids

Step 2: calculate the Euclidean distance from every node toward every centroid and affect it to the closest centroid.

By doing so, $\mathrm{k}$ initial clusters are produced

Step 3: Re-compute the positions of centroids inside every cluster by enchanting the mean of every data point (nodes) assign to that centroid's cluster.

$$
\text { new } w_{\text {center }}=\frac{1}{c_{i}} \sum_{j}^{c_{i}} s_{i}
$$

Where $c_{i}$ denoted the number of nodes in $i^{\text {th }}$ the cluster and $s_{i}$ represents sensor i

Step 4: If there is modification in any centroid's position afterwards, go to Step 2; else, the clusters are finalized along with the clustering process ends. 


\section{Algorithm 2: K-means++}

Input: A set $S=\left\{s_{1}, s_{2}, \ldots s_{n}\right\}$ of the sensor as well as a number $\mathrm{k}(\mathrm{k}>1)$ of clusters to form

Output: A set of k clusters generated

Step 1: Take one center $c_{i}$, preferred uniformly at random from $\mathrm{S}$

Step 2: Take second center $c_{i}$, equivalent to $s \varepsilon S$ by means of prospect $\frac{D(s)^{2}}{\sum D(s)^{2}}$

Step 3: replicate Step 2 until the k desired centers are taken.

Step 4: functional steps (2), (3) and (4) of standard K-Means to come to an end clustering process

The nodes transmit a packet with $\mu_{t p u t}$ rate using $E_{i}$ energy. The primary preferred position is to limit vitality, that is, utilization and boost network throughput. The models of these two destinations are spoken to in the accompanying subsections.

\section{A. Energy Consumption}

The energy consumed to transmit bundle among two hubs which are numerically spoken to as follows;

$$
\text { Energy }_{i}=2 E_{s t a r}+\frac{L}{R}\left(P_{t x}+P_{r x}+2 p_{c i r}+P_{a m p}\right)
$$

Where;

$E_{\text {star }}$ Corresponds to the amount of energy required to start the radio;

$P_{t x}$ And $P_{r x}$ is respectively the power consumption of the radio in transmission mode and receive mode;

$P_{c i r}$ is the electronic circuitry's power consumption;

L indicates the payload's bit size;

$\mathrm{R}$ reflects the data transfer rate;

$P_{\text {amp }}=\frac{(c d)^{n}}{B E R}$ denotes the energy consumption of the power amplifier, which is calculated by transmission range and BER (biterror-rate). $\mathrm{C}$ is a constant depending on channel attenuation and non-linear effect of the power amplifier; $\mathrm{d}$ is the transmission range, and $\mathrm{n}$ is the poss loss exponent;

\section{B. Packet throughput:}

Network throughput is the rate of the victorious packet that is conveyed over a correspondence channel. Along these lines, in the event that this boundary builds, the system proficiency will be expanded. This factor is influenced by two significant variables like bundle blunder rate and parcel length and is spoken to by:

$$
\mu_{\text {tput }}=\frac{L(1-P E R)}{T_{\text {flow }}}
$$

Where;

$P E R=1-(1-B E R)^{L}:$ Packet error rate.

$T_{\text {flow }}:$ is the end-to-end latency.

L: denotes the packet length 


\section{RESULTS AND DISCUSSION:}

In this part, a Hybrid TDR-MI Wireless Sensor Network is employed to identify and localize underground water pipeline leaks utilizing pressure residuals and classifiers. The proposed technique could be applied in MATLAB's workspace. This procedure is carried out on a Windows machine equipped with a dual-core processor, $1 \mathrm{~GB}$ of RAM, and a clock speed of $2.70 \mathrm{GHz}$ running the Microsoft Windows 7 Professional operating system.

\subsection{Performance metrics:}

The following measures are used to assess the performance of our suggested strategy. The performance characteristics of our hybrid TDR-MI technique are compared to those of linear, joint, and greedy algorithms.

Throughput: It is the amount of data transmitted per second from the sources to the destination. This parameter's unit is Mbps.

$$
\text { Throughput }=\frac{\text { Amount of transmitte d data }(M b)}{\text { Transmitte d time }(s)}
$$

Energy consumption: Each node consumes a certain amount of energy during transmission. Additionally, it is defined as the difference between a node's current and starting energy. This parameter's unit is the Joule (J).

$$
\text { Energy consumptio } n=\text { Initial energy - current energy }
$$

\subsection{Experimental results on proposed approach:}

The main idea of our proposed methodology is underground water pipeline leakage detection and leak localization based on hybrid TDR-MI. The proposed result is compared with existing linear, joint as well as greedy algorithms. The following figure 6 to 9 shows the performance of the proposed approach using this configuration,

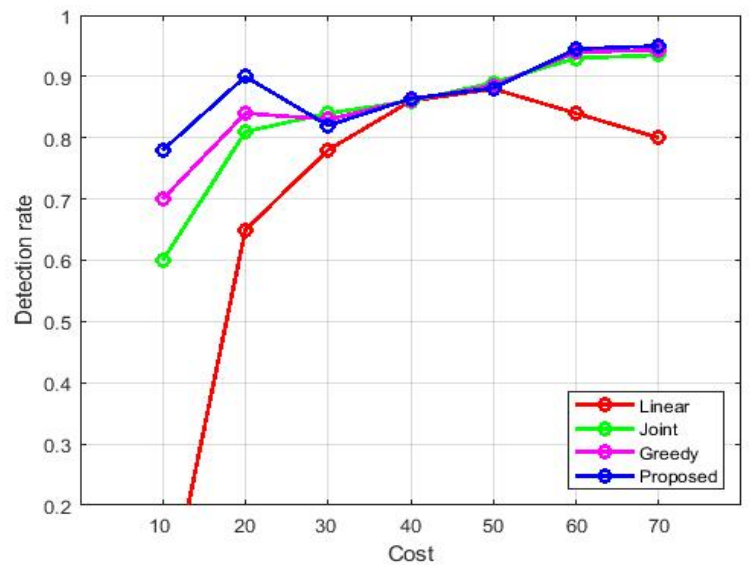

6(a). Detection rate

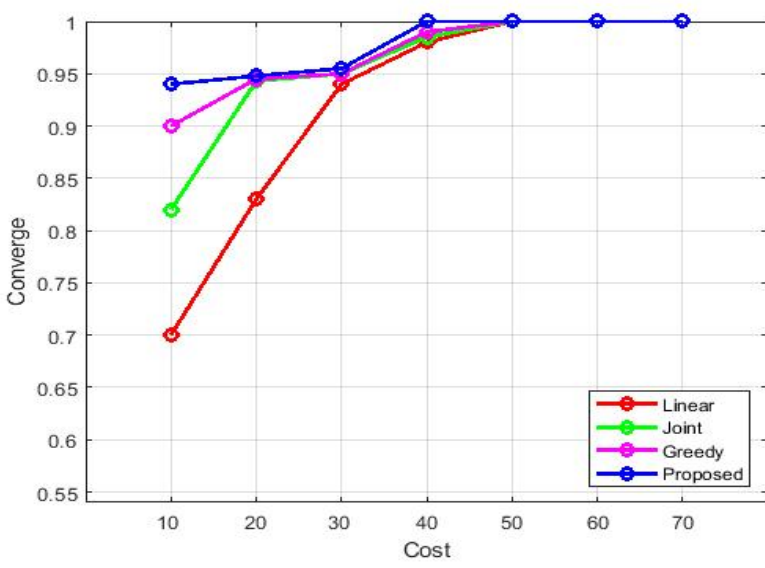

6(b) Converge

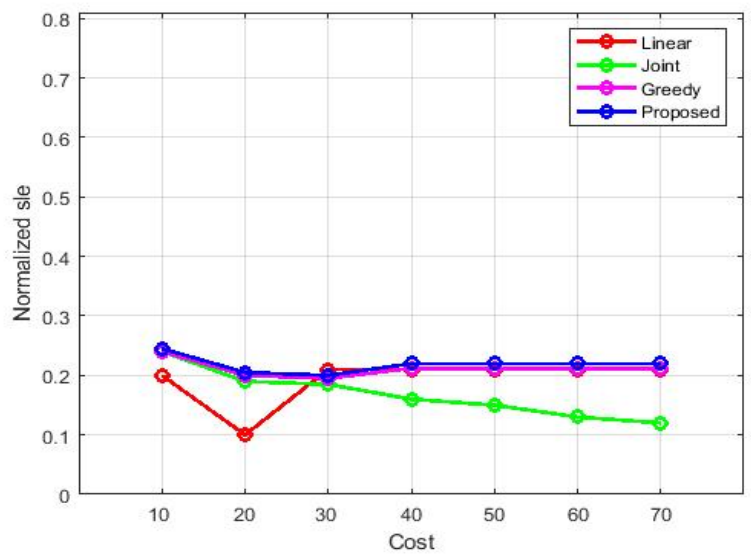

6(c) Normalized SLE 
Figure 6 shows the presentation investigation of proposed against existing utilizing cost work. Here, our proposed approach used the least expense to accomplish the objective. Fig. 6 delineates the affectability of execution measurements to the passageway reference point sensor cost proportion. The cost spending plan is set to be 50 . Standardized SLE expands by and large with expanding the cost proportion. This is on the grounds that less reference points are put. Fewer guides lead to expanded brew because there are more funnels between every signal set. When the cost proportion is 2, the joint plan places reference points at all vertices, saving money on sensors, determined to be the optimal solution for that cost percentage. This results in a higher rate of inclusion and a lower SLE for the cost ratio of three. Be that as it may, the crossbreed TDR-MI plan can keep up an expanding pattern in SLE because ordinarily, it advances the standard inclusion and limitation blunders. Over a cost proportion of 5 , the pattern is increasingly uniform overall details. Figure 6 unmistakably comprehend our proposed approach accomplishes the better outcomes contrast with existing methodologies.
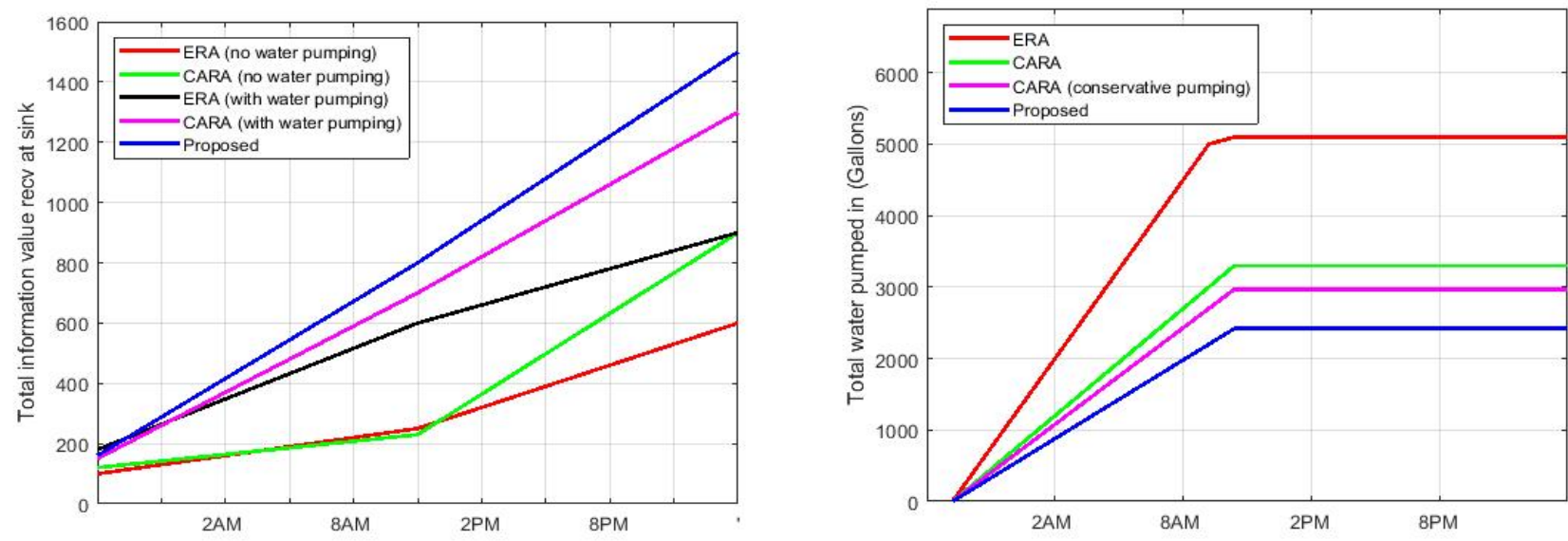

Figure 7: Comparison of proposed and current energy-efficient technologies

Figure 7 shows the exhibition examination of proposed against existing utilizing vitality work. Our proposed approach used to ascertain complete data esteem got at the sink and absolute water siphoned in (gallons) to accomplish the objective. Figure 7 comprehend that our proposed approach performs better outcomes in contrast with existing methodologies.

8(a) Detection

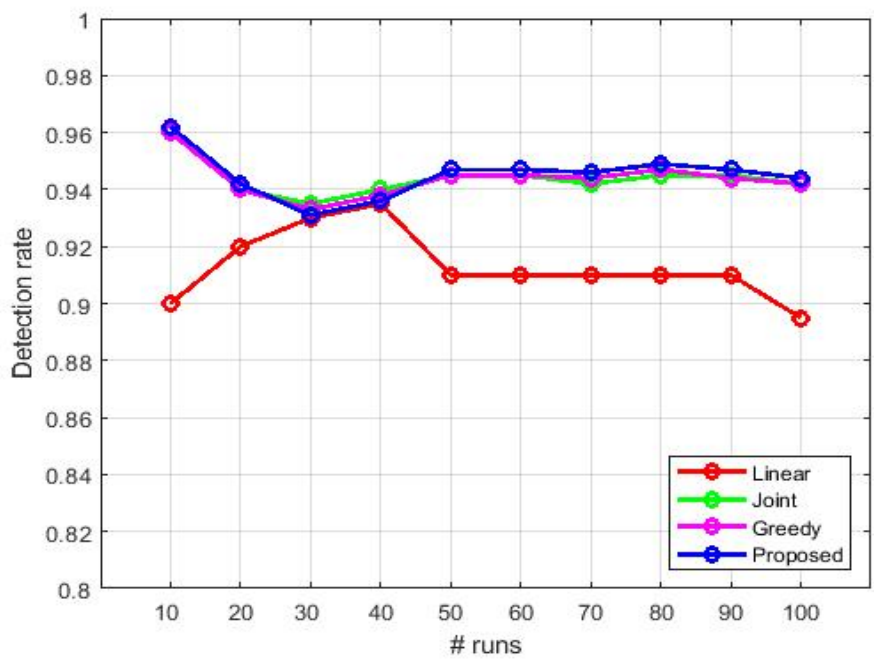




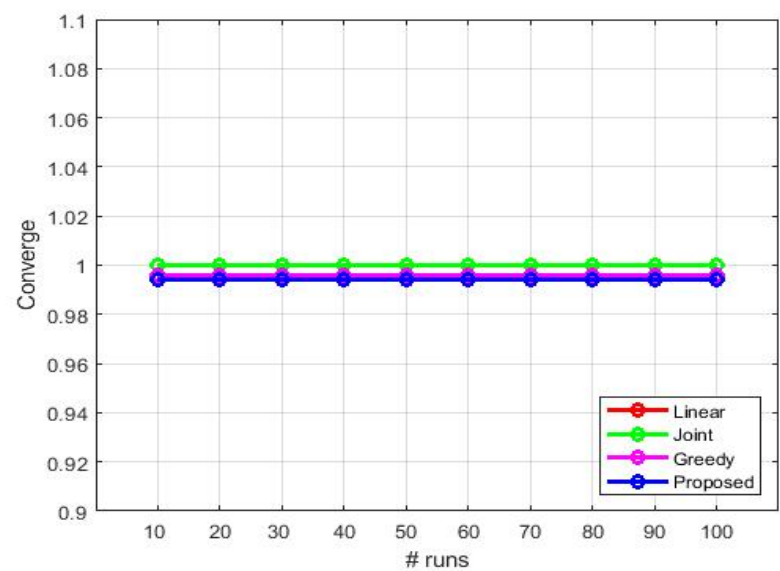

8(b) Coverage

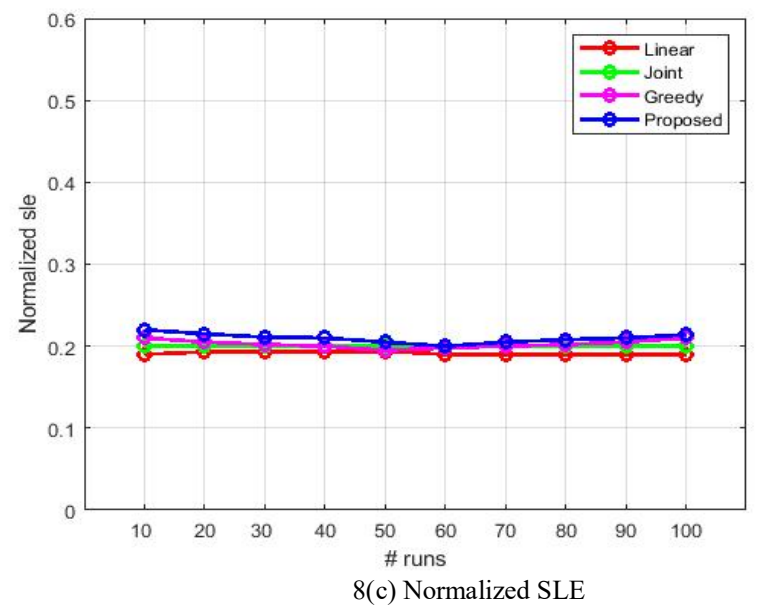

Figure 8: Performance comparison of suggested against existing technologies utilizing a variety of simulation runs for (a) Detection rate, (b) Coverage, and (c) normalized SLE

The obtained coverage, discovery rate, and SLE values for various run counts are shown in Fig. 8. The cost financial plan is set to 50. In any case, the blunder bars for inclusion, bit by bit, diminish. If the standardised SLE should arise, the blunder bars are broad because the edge restriction mistakes are diverse for each edge. In addition, if the break isn't identified, the standardized SLE is 1, along these lines expanding the difference in the outcomes. Figure 8 unmistakably comprehends that our proposed approach accomplishes better results in contrast with existing methodologies.
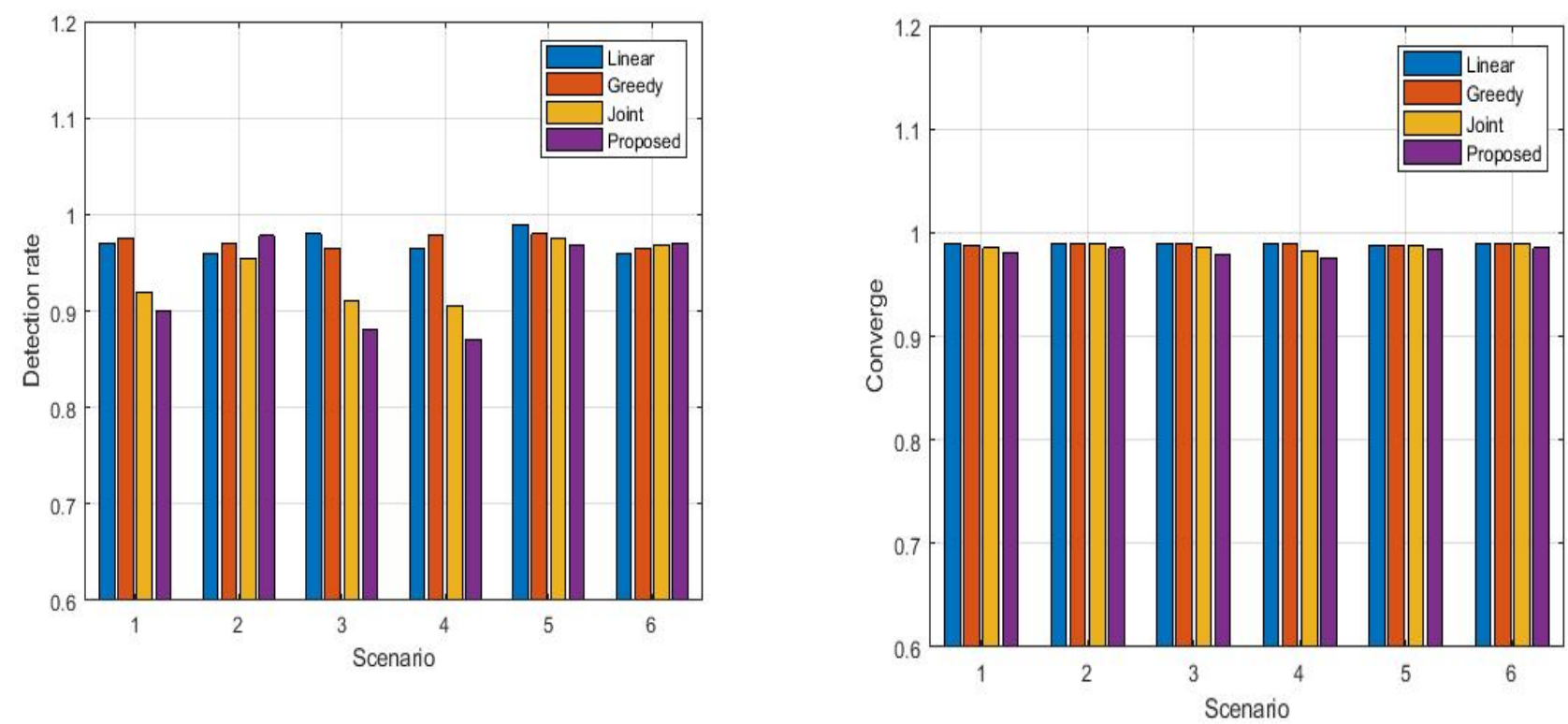

9( a) Detection rate and 9 ( b ) Converge 


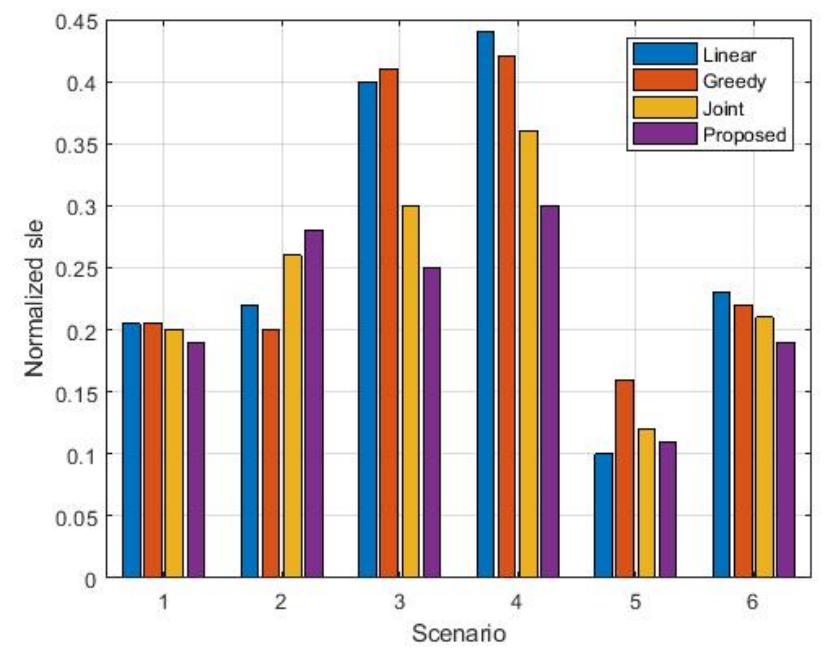

9( c ) normalize SLE

Figure 9: Performance analysis of proposed against existing using, when the location of beacons and sensors may be restricted for (a) Detection rate, (b) Coverage, and (c) normalized SLE

We test the robustness of our answer by adding limitations to the position of reference points and sensors, as appeared in Fig. 9. The cost financial plan is set to be 50. Confining the position of reference points and sensors hurts the inclusion and identification rate true to form. In any case, with a more significant expense, the signal situation limitation influences SLE more than the sensor arrangement limitation. It is seen that when both sensor and reference point arrangement is limited harshly, the guides are sent at all conceivable vertices, even though it decreases the number of sensors marginally when contrasted with a situation when there are fewer limitations. Figure 9 comprehend that our proposed approach accomplishes better outcomes in contrast with existing methodologies.

\section{CONCLUSION}

This research proposes a hybrid TDR-MI-based wireless sensor network architecture for underground pipeline monitoring (MISEPIPE). This configuration is capable of detecting underground pipe leaks. MISE-PIPE is equipped with distributed Bi-Wired technology and incorporates sensors both within and outside the pipes, such as ad-hoc wireless sensor networks for pressure monitoring, acoustic sensors, and soil quality sensors. These sensors work in tandem to detect pipeline leaks underneath. The unique hybrid magnetic induction technique offers wireless communication for subsurface sensors that are both efficient and robust. The location of a leak is determined using pressure residuals and a statistical classifier. Following detection and localization of pipeline leaks, all monitoring data is forwarded to the $\mathrm{CH}$ using the K-means clustering algorithm. Our proposed Hybrid TDR-MI-based wireless sensor network enables accurate real-time leak identification and location compared to the present underground pipeline monitoring system.

\section{DECLARATIONS}

This work was supported by the Department of Computer Science and Engineering, University College of Engineering(A), Osmania University, Hyderabad-500007, India.

\section{REFERENCE}

[1] Tarek R. Sheltami, AbubakarBala, "Wireless sensor networks for leak detection in pipelines: a survey," Springer-J Ambient Intell Human Comput, 10 January 2016.

[2] A. Gupta and K. D. Kulat, "A selective literature review on leak management techniques for the water distribution system," Springer-Water Resource. Manage., vol. 32, no. 10, pp. 3247-3269, Aug. 2018.

[3] S. Datta and S. Sarkar, "A review on different pipeline fault detection methods," J. Loss Prevention Process Ind., vol. 41, pp. 97-106, May 2016.

[4] M. Zadkarami, M. Shahbazian, and K. Salahshoor, "Pipeline leak diagnosis based on wavelet and statistical features using Dempster-Shafer classifier fusion technique," Process Saf. Environ. Protection, vol. 105, Jan. 2017.

[5] Kayaalp, F., Zengin, A., Kara, R., Zavrak, S.: Leakage detection and localization on water transportation pipelines: a multi-label classification approach. Neural Comput.Appl. 2017. 
[6] K. Adedeji, Y. Hamam, B. Abe, and A. M. Abu-Mahfouz, "Pressure management strategies for water loss reduction in large-scale water piping networks: A review," in Proc. Choosing Right Model Appl. Hydraulics (SimHydro), Sophia Antipolis, France, Jun. 2017.

[7] MortezaZadkarami, Mehdi Shahbazian, "Pipeline leak diagnosis based on wavelet and statistical features using Dempster-Shafer classifier fusion technique," Elsevier- Department of Instrumentation and Automation, Petroleum University of Technology, November 2016.

[8] W. Lu, L. Zhang, W. Liang, and X. Yu, "Research on a small-noise reduction method based on EMD and its application in pipeline leakage detection," J. Loss Prevention Process Ind., vol. 41, May 2016.

[9] Haoran Zhang, Yongtu Liang, "Improved PSO-based Method for Leak Detection and Localization in Liquid Pipelines," IEEE Transactions on Industrial Informatics, 2017

[10]Thaw Tar TheinZan, Hock Beng Lim, "Event Detection and Localization in Urban Water Distribution Network," IEEE SENSORS JOURNAL, VOL. 14, NO. 12, DECEMBER 2014.

[11] Y. Guo, K. Huang, N. Jiang, X. Guo, Y. Li, and G. Wang, "An Exponential-Rayleigh Model for RSS-Based Device-Free Localization and Tracking," IEEE Transactions on Mobile Computing, vol. 14, no. 3, Mar. 2015.

[12] Haoran Zhang, Yongtu Liang, "Improved PSO-based Method for Leak Detection and Localization in Liquid Pipelines," IEEE Transactions on Industrial Informatics, 2017.

[13] Dalei Wu, DimitrisChatzigeorgiou, "Node Localization in Robotic Sensor Networks for Pipeline Inspection," IEEE Transactions on Industrial Informatics, 2015.

[14] Chao-Chih Lin, "A Hybrid Heuristic Optimization Approach for Leak Detection in Pipe Networks Using Ordinal Optimization Approach and the Symbiotic Organism Search," Institute of Environmental Engineering, 2017.

[15] LiseFerrandez-gamot, Pierre Busson, "peak Localization in water distribution networks using pressure residuals and classifiers," ELSEVIER 2015.

[16] Xue-yuan WANG, Zhi-gang CHEN, "Research on Leak Detection of Water Pipeline Base on PSO-SVM," Electronics and Mechatronics Engineering, 2016.

[17] ZiguangJia, Siu-Chun Ho, "Multipoint hoop strain measurement based pipeline leakage localization with an optimized support vector regression approach," Elsevier Journal of Loss Prevention in the Process Industries, 2019.

[18] AirullAziziAwangLah*, RudzidatulAkmamDziyauddin, "Localization Techniques for Water Pipeline Leakages: A Review," 30 November 2018.

[19] Hakiri, Akram, Pascal Berthou, AniruddhaGokhale, and Slim Abdellatif. "Publish/subscribe-enabled software-defined networking for efficient and scalable IoT communications." IEEE communications magazine 53, no. 9 (2015): 48-54.

[20] Alanazi, Shaker, Jalal Al-Muhtadi, AbdelouahidDerhab, KashifSaleem, Afnan N. AlRomi, Hanan S. Alholaibah, and Joel JPC Rodrigues. "On the resilience of Wireless Mesh routing protocol against DoS attacks in IoT-based ambient assisted living applications" In 2015 17th International Conference on E-health Networking, Application \& Services (HealthCom), pp. 205-210, IEEE, 2015.

[21] Sung, Wen-Tsai, Jui-Ho Chen, and Ming-Han Tsai, "Applications of wireless sensor network for monitoring system based on IoT," In 2016 IEEE international conference on systems, man, and cybernetics (SMC), pp. 000613-000617, IEEE, 2016.

[22] Lee, Huang-Chen, and Kai-Hsiang Ke. "Monitoring of large-area IoT sensors using a LoRa wireless mesh network system: Design and evaluation." IEEE Transactions on Instrumentation and Measurement 67, no. 9 (2018): 2177-2187.

[23] Granjal, Jorge, EdmundoMonteiro, and Jorge Sá Silva. "Security in the integration of low-power Wireless Sensor Networks with the Internet: A survey." Ad Hoc Networks 24 (2015): 264-287.

[24] Kharrufa, Harith, Hayder Al-Kashoash, Yaarob Al-Nidawi, Maria Quezada Mosquera, and Andrew H. Kemp. "Dynamic RPL for multi-hop routing in IoT applications" In 2017 13th Annual Conference on Wireless On-demand Network Systems and Services (WONS), pp. 100-103, IEEE, 2017.

[25] Da Costa, Gustavo A., and João H. Kleinschmidt "Implementation of a wireless sensor network using standardized IoT protocols" In 2016 IEEE International Symposium on Consumer Electronics (ISCE), pp. 17-18, IEEE, 2016.

[26] Nair, Karan, JanhaviKulkarni, MansiWarde, Zalak Dave, VedashreeRawalgaonkar, Ganesh Gore, and Jonathan Joshi. "Optimizing power consumption in IoT based wireless sensor networks using Bluetooth Low Energy" In 2015 International Conference on Green Computing and Internet of Things (ICGCIoT), pp. 589-593, IEEE, 2015.

[27] Mainetti, Luca, Luigi Patrono, and Antonio Vile. "Evolution of wireless sensor networks towards the internet of things: A survey." In SoftCOM 2011, 19th international conference on software, telecommunications and computer networks, pp. 1-6, IEEE, 2011.

[28] Ramdas Vankdothu, Mohd Abdul Hameed "Effective Localization of Energy Harvesting Empowered UWSNS Using Krill Herd Optimization and Support Value" under review on IEEE Transaction on Green Communication and Networking.

[29] Ramdas Vankdothu, Mohd Abdul "Energy Efficient TDMA and Secure based MAC Protocol for WSN using AQL Coding and ASGWI clustering" under review on IEEE Transaction on Green Communication and Networking.

[30] Ramdas Vankdothu, Mohd Abdul "Multicast Scaling in Heterogeneous Wireless Sensor Networks for Security and Time Efficiency" under review on IEEE Transaction on wireless communication.. 